\title{
Diagonal and off-diagonal types of disorder in condensed matter theory
}

\author{
Yu.Rudavskii, G.Ponedilok, Yu.Petriv \\ Lviv National Polytechnic University \\ 12 S.Bandera Str., 79013 Lviv, Ukraine
}

Received August 28, 2000, in final form November 1, 2000

\begin{abstract}
In the paper we investigate the distribution function of a diagonal type of disorder, their relation with microscopic model parameters and structural correlation functions. The conditions are obtained for which the distribution of random values of atomic energy levels will be of Gauss type. The numerical analysis of dispersion $\mathcal{D}$ is performed. The distribution to the hydrogen-like atom chemisorption on an amorphous system is applied.
\end{abstract}

Key words: amorphous systems, structural functions, diagonal disorder, off-diagonal disorder

PACS: $73.20 . H, 82.65 . Y$

\section{Introduction}

In the theory of structurally disordered systems, traditionally diagonal and offdiagonal types of disorder [1] are used. In particular, the diagonal type of disorder describes fluctuations of one-cite parameters (energies of electronic atom levels, random magnetic or electric field on atom etc.), and off-diagonal disorder describes random transfer integral values, or other two-cite descriptions. Often diagonal and off-diagonal disorder types are used together in one model and can bring some unconformity.

In the theory of amorphous systems, disorder is determined by random atoms position, configurations of which are described by correlation structural functions. Diagonal disorder is caused by random atoms configuration. In some studies, namely in [2-3], equilibrium distribution functions of some models are calculated. Also, very important is the problem of the correct definition of disorder correlation functions in the problem of Anderson localization [4]. However, different model distribution functions of diagonal random parameters for systems are often used (regular distribution, Lorenz distribution, Gauss distribution etc.).

In the paper we investigate distribution functions of diagonal disorder, their relations with microscopic model parameters and structural correlation functions. In 
section 2 the model of random media with the probe atom are considered. There is shown the physical mechanism of diagonal disorder type and in section 3 distribution functions are obtained in some approximation. In the structurally disordered system atoms position are random. The total effective potential, which is created by randomly located atoms in some media point, is the random value. This potential is of Stark type and shifts electronic levels of atoms. That position of the levels in the dependence on the localization point of the probe atom, is the random value as well. Finally, the obtained results are discussed in conclusions.

\section{The model of disordered media}

Let us consider a microscopic model of structurally disordered media: the system of $N$ atoms, which are located in positions $\mathbf{R}_{1}, \ldots, \mathbf{R}_{N}$ in the volume $V$ and the impurity atom in $\mathbf{R}_{0}$ position. The initial Hamiltonian of the model in the coordinate image is as follows:

$$
\begin{aligned}
\hat{H}= & -\frac{\hbar^{2}}{2 m} \sum_{1 \leqslant i \leqslant N_{e}} \Delta_{i}+\sum_{1 \leqslant i \leqslant N_{e}} \sum_{1 \leqslant j \leqslant N} V\left(\left|\mathbf{r}_{i}-\mathbf{R}_{j}\right|\right) \\
& +\sum_{1 \leqslant i \leqslant N_{e}} V_{0}\left(\left|\mathbf{r}_{i}-\mathbf{R}_{0}\right|\right)+\frac{1}{2} \sum_{1 \leqslant i \neq j \leqslant N_{e}} W\left(\left|\mathbf{r}_{i}-\mathbf{r}_{j}\right|\right) .
\end{aligned}
$$

Here $V\left(\left|\mathbf{r}-\mathbf{R}_{\mathbf{j}}\right|\right)$ and $V_{0}\left(\left|\mathbf{r}-\mathbf{R}_{\mathbf{0}}\right|\right)$ are electron potential energies in the field of atom with coordinate $\mathbf{R}_{j}$ and in the field of impurity $\mathbf{R}_{0}$, respectively. The value $W\left(\left|\mathbf{r}_{i}-\mathbf{r}_{j}\right|\right)$ describes the energy of Coulomb electrons interaction.

The solutions $\left\{\Psi_{\mu}\left(\mathbf{r}-\mathbf{R}_{j}\right), \mathcal{E}_{\mu}\right\}$ of Schrodinger equation in the field $V\left(\left|\mathbf{r}-\mathbf{R}_{\mathbf{j}}\right|\right)$ and the solution $\left\{\varphi_{\mu}\left(\mathbf{r}-\mathbf{R}_{0}\right), E_{\mu}\right\}$ of Schrodinger equation in the field $V_{0}\left(\left|\mathbf{r}-\mathbf{R}_{\mathbf{0}}\right|\right)$ are known. The functions $\Psi_{\mu}\left(\mathbf{r}-\mathbf{R}_{j}\right)$ and $\varphi_{\mu}\left(\mathbf{r}-\mathbf{R}_{0}\right)$ are used as the basis for expansion of field operators. For simplicity we use as a basis only valency $s$-orbitals of the atoms. For such orbitals notations $\Psi\left(\mathbf{r}-\mathbf{R}_{j}\right)$ and $\varphi\left(\mathbf{r}-\mathbf{R}_{0}\right)$, the respective atom levels $\mathcal{E}_{0}$ and $E_{0}$ are employed. The problems of incompleteness of such Gilbert space basis and physical consequences of it are well familiar and they are not discussed here.

The Hamilton operator of disordered media in secondary quantization representation is

$$
\hat{H}=C+\hat{H}_{0}+\hat{H}_{\text {corr }} .
$$

The non-operator part of Hamiltonian (2)

$$
C=-U_{0}\left\langle\hat{n}_{0 \uparrow}\right\rangle\left\langle\hat{n}_{0 \downarrow}\right\rangle-\sum_{1 \leqslant j \leqslant N} U\left\langle\hat{n}_{j \uparrow}\right\rangle\left\langle\hat{n}_{j \downarrow}\right\rangle-\sum_{1 \leqslant j \leqslant N} \Phi\left(\left|\mathbf{R}_{j}-\mathbf{R}_{0}\right|\right)\left\langle\hat{n}_{j}\right\rangle\left\langle\hat{n}_{0}\right\rangle
$$

is electron-electron effective electrostatic interactions. Values $\left\langle\hat{n}_{j}\right\rangle=\left\langle\hat{n}_{j \uparrow}\right\rangle+\left\langle\hat{n}_{j \downarrow}\right\rangle$, $\left\langle\hat{n}_{0}\right\rangle=\left\langle\hat{n}_{0 \uparrow}\right\rangle+\left\langle\hat{n}_{0 \downarrow}\right\rangle$ are the thermodynamically averaged number of electrons, localized on $j$-th atom and on impurity atom, respectively. Hereinafter the symbol $\langle\ldots\rangle$ means the average over a grand canonical ensemble. 
The effective one-particle Hamiltonian of the model is

$$
\begin{aligned}
\hat{H}_{0}= & \sum_{1 \leqslant j \leqslant N} \sum_{\sigma=\uparrow, \downarrow} \mathcal{E}_{j, \sigma} a_{j \sigma}^{+} a_{j \sigma}+\sum_{\sigma=\uparrow, \downarrow} E_{\sigma} d_{0 \sigma}^{+} d_{0 \sigma}+\sum_{1 \leqslant i \neq j \leqslant N} \sum_{\sigma=\uparrow, \downarrow} T_{i j} a_{i \sigma}^{+} a_{j \sigma} \\
& +\sum_{1 \leqslant j \leqslant N} \sum_{\sigma=\uparrow, \downarrow}\left(V_{j, 0} a_{i \sigma}^{+} d_{0 \sigma}+V_{0, j} d_{0 \sigma}^{+} a_{i \sigma}\right) .
\end{aligned}
$$

Here $a_{j \sigma}\left(a_{j \sigma}^{+}\right)$and $d_{0 \sigma}\left(d_{0 \sigma}^{+}\right)$are Fermi annihilation (creation) operators of electrons on $\mathbf{R}_{j}$-th atom and on $\mathbf{R}_{0}$ impurity atom, respectively. Further in the paper we employed notations $\hat{n}_{j, \sigma}=a_{j \sigma}^{+} a_{j \sigma}$ and $\hat{n}_{0, \sigma}=d_{0 \sigma}^{+} d_{0 \sigma}$ for the operator of number of electrons in localized atom states, and both $\hat{n}_{j}=\hat{n}_{j, \uparrow}+\hat{n}_{j, \downarrow}$ and $\hat{n}_{0}=\hat{n}_{0, \uparrow}+\hat{n}_{0, \downarrow}$ for the operator of the total number of electrons.

The operator of electron-electron correlations in Hamiltonian (2) is

$$
\begin{aligned}
\hat{H}_{\text {corr }}= & \frac{U_{0}}{2} \sum_{\sigma=\uparrow, \downarrow} \delta \hat{n}_{0 \sigma} \delta \hat{n}_{0,-\sigma}+\frac{U}{2} \sum_{1 \leqslant j \leqslant N} \sum_{\sigma=\uparrow, \downarrow} \delta \hat{n}_{j \sigma} \delta \hat{n}_{j,-\sigma} \\
& +\sum_{1 \leqslant j \leqslant N} U\left(\left|\mathbf{R}_{j}-\mathbf{R}_{0}\right|\right) \delta \hat{n}_{j} \delta \hat{n}_{0} .
\end{aligned}
$$

In this expression we employed notes $\delta \hat{n}_{j \sigma}=\hat{n}_{j \sigma}-\left\langle\hat{n}_{j \sigma}\right\rangle$, and $\delta \hat{n}_{0 \sigma}=\hat{n}_{0 \sigma}-\left\langle\hat{n}_{0 \sigma}\right\rangle$ for operators of fluctuations of the number of electrons, localized on the atoms in the point of their averaged thermodynamic sense. The parameter $U$ is the energy of Hubbard interaction between electrons, which are localized on $s$-orbital media atom. The $U_{0}$ is the energy of Hubbard interaction between electrons, which are localized on the impurity $s$-orbital.

The renormalized energy of localized level on $\mathbf{R}_{j}$-th atom and on the impurity atom has the following structure

$$
\begin{aligned}
\mathcal{E}_{j, \sigma} & =\mathcal{E}_{0}+U\left\langle\hat{n}_{j,-\sigma}\right\rangle+\Delta \mathcal{E}_{j}, \\
E_{\sigma} & =E_{0}+U_{0}\left\langle\hat{n}_{0,-\sigma}\right\rangle+\Delta E .
\end{aligned}
$$

Here media and impurity atom level shift, respectively are

$$
\begin{aligned}
\Delta \mathcal{E}_{j}= & \Phi_{0}\left(\left|\mathbf{R}_{j}-\mathbf{R}_{0}\right|\right)+U_{0}\left(\left|\mathbf{R}_{j}-\mathbf{R}_{0}\right|\right)\left\langle\hat{n}_{0}\right\rangle \\
& +\sum_{i=1(i \neq j)}^{N}\left[\Phi\left(\left|\mathbf{R}_{j}-\mathbf{R}_{i}\right|\right)+U\left(\left|\mathbf{R}_{j}-\mathbf{R}_{i}\right|\right)\left\langle\hat{n}_{i}\right\rangle\right], \\
\Delta E= & \sum_{j=1}^{N}\left[\Phi\left(\left|\mathbf{R}_{0}-\mathbf{R}_{j}\right|\right)+U_{0}\left(\left|\mathbf{R}_{0}-\mathbf{R}_{j}\right|\right)\left\langle\hat{n}_{j}\right\rangle\right] .
\end{aligned}
$$

In these expressions the parameter $\Phi_{0}\left(\left|\mathbf{R}_{j}-\mathbf{R}_{0}\right|\right)$ describes the $j$-th atom level shift under impurity pseudopotential effect

$$
\Phi_{0}\left(\left|\mathbf{R}_{j}-\mathbf{R}_{0}\right|\right)=\int \Psi^{2}\left(\mathbf{r}-\mathbf{R}_{j}\right) V_{0}\left(\left|\mathbf{r}-\mathbf{R}_{0}\right|\right) \mathrm{d} \mathbf{r} .
$$


Here $V_{0}\left(\left|\mathbf{r}-\mathbf{R}_{0}\right|\right)$ is pseudopotential of impurity. The electron effective energy in the $\mathbf{R}_{j}$-th atom potential field is

$$
\begin{aligned}
\Phi\left(\left|\mathbf{R}_{0}-\mathbf{R}_{j}\right|\right) & =\int \varphi^{2}\left(\mathbf{r}-\mathbf{R}_{0}\right) V\left(\left|\mathbf{r}-\mathbf{R}_{j}\right|\right) \mathrm{d} \mathbf{r} \\
& =V \frac{1}{R_{0 j}} \frac{\mathrm{e}^{-2 \kappa R_{0 j}}-\mathrm{e}^{-2 R_{0 j}}\left[1-\kappa^{2} R_{0 j}+R_{0 j}\right]}{(\kappa-1)^{2}(\kappa+1)^{2}}
\end{aligned}
$$

where $V=-\left(-Z e^{2}\right) / a_{0}, R_{0 j}=\left|\mathbf{R}_{0}-\mathbf{R}_{j}\right|$ and $a_{0}$ is the Bohr radius of the hydrogen atom. Here we used the model pseudopotential

$$
V\left(\left|\mathbf{r}-\mathbf{R}_{j}\right|\right)=-\frac{-Z \mathrm{e}^{2}}{\left|\mathbf{r}-\mathbf{R}_{j}\right|} \mathrm{e}^{-2 \kappa\left|\mathbf{r}-\mathbf{R}_{j}\right|}
$$

where $Z$ is the effective charge value of the ions and the parameter $\kappa>0$.

The Coulomb integral

$$
U_{0}\left(\left|\mathbf{R}_{0}-\mathbf{R}_{j}\right|\right)=\iint \varphi^{2}\left(\mathbf{r}-\mathbf{R}_{0}\right) W(|\mathbf{r}-\mathbf{r} \prime|) \Psi^{2}\left(\mathbf{r}^{\prime}-\mathbf{R}_{j}\right) \mathrm{d} \mathbf{r} \mathrm{d} \mathbf{r}^{\prime}
$$

is the averaged interaction energy between electrons, one of which is localized on $\Psi\left(\mathbf{r}-\mathbf{R}_{j}\right)$ orbital of media atom, and the second is localized on $\varphi\left(\mathbf{r}-\mathbf{R}_{0}\right)$ impurity orbital. For $s$-orbital, this energy depends only on the distance between atoms. The values $\Delta \mathcal{E}_{j}$ and $\Delta E$ are random, because the atoms configuration $\mathbf{R}_{1}, \ldots, \mathbf{R}_{n}$ of an amorphous system is also random. The distribution function of $\Delta E$ random value in the next section will be obtained.

\section{Distribution function of off-diagonal and diagonal disorder}

In the case of amorphous media, the atoms configuration is convinient to be described by collective variables [5]

$$
\rho_{\mathbf{k}}=\frac{1}{\sqrt{N}} \sum_{j=1}^{N} \mathrm{e}^{-i \mathbf{k} \mathbf{R}_{j}}, \quad \mathbf{k} \neq \mathbf{0}
$$

Here $\mathbf{k} \in \Lambda$ note the wave vector, which takes values in a quasi continuous impulse space $\Lambda$ :

$$
\Lambda=\left\{\mathbf{k}: \mathbf{k}=\sum_{\alpha=x, y, z} 2 \pi V^{-1 / 3} n_{\alpha} \mathbf{e}_{\alpha}, \quad n_{\alpha} \in \mathbb{Z}, \quad\left(\mathbf{e}_{\alpha}, \mathbf{e}_{\beta}\right)=\delta_{\alpha \beta}\right\}
$$

The structure of amorphous media is described by correlation functions

$$
S_{m}\left(\mathbf{k}_{1}, \ldots, \mathbf{k}_{m}\right) \delta_{\mathbf{k}_{1}+\ldots+\mathbf{k}_{m}, \mathbf{0}} \stackrel{\text { def }}{=}\left[\frac{1}{\sqrt{N}}\right]^{2-m}\left\langle\rho_{\mathbf{k}_{1}} \ldots \rho_{\mathbf{k}_{m}}\right\rangle_{\mathbf{R}}^{\text {irr }}
$$


which are the irreducible averaged by the configuration from the product of collective variables. The operation of the configuration averaging enabled us to define in the following way

$$
\langle(\ldots)\rangle_{\mathbf{R}}=\int\left(\mathrm{d} \rho_{\mathbf{k}}\right) P\left(\ldots, \rho_{\mathbf{k}}, \ldots\right)(\ldots) .
$$

Density fluctuation probabilities distribution function are approximated by Gauss law

$$
P\left(\ldots, \rho_{\mathbf{k}}, \ldots\right)=\prod_{\mathbf{k} \neq \mathbf{0}}\left[\frac{1}{\sqrt{2 \pi S_{2}(k)}}\right] \exp \left[-\sum_{\mathbf{k} \neq \mathbf{0}} \frac{\rho_{\mathbf{k}} \rho_{-\mathbf{k}}}{S_{2}(k)}\right] .
$$

Dispersion of Gauss distribution fluctuations

$$
S_{2}(k)=\int\left(\mathrm{d} \rho_{\mathbf{k}}\right) \rho_{\mathbf{k}} \rho_{-\mathbf{k}} P\left(\ldots, \rho_{\mathbf{k}}, \ldots\right)
$$

is the pair structural factor of the system. In the present model $S_{2}(k)$ is the model function, which contains adjustment parameters. Integration symbol for collective variables is as follows:

$$
\int\left(\mathrm{d} \rho_{\mathbf{k}}\right)(\ldots)=\prod_{\mathbf{k}} \int_{-\infty}^{+\infty} \mathrm{d} \operatorname{Re} \varrho_{\mathbf{k}} \int_{-\infty}^{+\infty} \mathrm{d} \operatorname{Im} \varrho_{\mathbf{k}}(\ldots)
$$

where $\varrho_{\mathbf{k}}=\operatorname{Re} \varrho_{\mathbf{k}}-\mathrm{i} \operatorname{Im} \varrho_{\mathbf{k}}$. The real and the imaginary parts of collective variables satisfy the conditions that $\operatorname{Re} \rho_{\mathbf{k}}=\operatorname{Re} \rho_{-\mathbf{k}}$ and $\operatorname{Im} \rho_{\mathbf{k}}=-\operatorname{Im} \rho_{-\mathbf{k}}$.

Energy of localized level of impurity (9) depends on a random configuration of the atoms. By neglecting the values of a higher order for density fluctuations $\varrho_{\mathbf{k}}$, the expression (9) may be rewritten in the form:

$$
E_{\sigma}=\bar{E}_{\sigma}+\Delta\left[\varrho_{\mathbf{k}}\right]
$$

The level energy independent on the random configurations is

$$
\bar{E}_{\sigma}=E_{0}+U_{0}\left\langle\hat{n}_{0,-\sigma}\right\rangle+\frac{N}{V}\left(\widetilde{\Phi}_{0}+\bar{n} \widetilde{U}_{0}\right) .
$$

The fluctuation part of $E_{\sigma}$ as a function of $\varrho_{\mathbf{k}}$ may be written in the form:

$$
\Delta\left[\varrho_{\mathbf{k}}\right]=\frac{\sqrt{N}}{V} \sum_{\mathbf{k} \neq \mathbf{0}}\left(\widetilde{\Phi}_{k}+\bar{n} U_{k}\right) \varrho_{-\mathbf{k}} \mathrm{e}^{-\mathrm{i} \mathbf{k} \mathbf{R}_{0}}
$$

Here $\bar{n}=N^{-1} \sum_{j=1}^{N}\left\langle\hat{n}_{j}\right\rangle$ is the average thermodynamic number of electrons, localized on the atom orbitals in computation per one atom. The coefficients $\widetilde{\Phi}_{k} \quad \widetilde{U}_{k}$ are Fourier-components of Fourier series expansion of the effective energies of electronelectron interactions

$$
\Phi_{s}\left(\left|\mathbf{R}_{0}-\mathbf{R}_{j}\right|\right)=\frac{1}{V} \sum_{\mathbf{k}} \widetilde{\Phi}_{k} \mathrm{e}^{\mathrm{i} \mathbf{k}\left(\mathbf{R}_{0}-\mathbf{R}_{j}\right)}, \quad U_{k}\left(\left|\mathbf{R}_{0}-\mathbf{R}_{j}\right|\right)=\frac{1}{V} \sum_{\mathbf{k}} \widetilde{U}_{k} \mathrm{e}^{\mathrm{ik}\left(\mathbf{R}_{0}-\mathbf{R}_{j}\right)} .
$$


The inverse transformations are

$$
\widetilde{\Phi}_{k}=\int_{V} \Phi_{s}(\mathbf{R}) \mathrm{e}^{\mathrm{i} \mathbf{k} \mathbf{R}} d \mathbf{R}, \quad \widetilde{U}_{k}=\int_{V} U(\mathbf{R}) \mathrm{e}^{-\mathrm{i}(\mathbf{k} \mathbf{R}} d \mathbf{R} .
$$

For a diagonal type of disorder, the energy probability distribution function of localized states can be calculated by the formula

$$
P\left(E_{\sigma}\right)=\left\langle\delta\left(E_{\sigma}-E_{\sigma}\{\mathbf{R}\}\right\rangle_{\mathbf{R}}\right.
$$

Using the expansion of $\delta$-function in Fourier series and distribution function $P\left(\ldots, \varrho_{\mathbf{k}}, \ldots\right)$, given by formula $(15)$, for $P\left(E_{\sigma}\right)$, we obtained

$$
P\left(E_{\sigma}\right)=\frac{1}{\sqrt{2 \pi \mathcal{D}}} \exp \left(-\frac{\left(E_{\sigma}-\bar{E}_{\sigma}\right)^{2}}{2 \mathcal{D}}\right),
$$

where the dispersion of distribution is

$$
\mathcal{D}=\frac{1}{V} \sum_{\mathbf{k}}\left(\widetilde{\Phi}_{k}+\bar{n} U_{k}\right)^{2} S_{2}(k)
$$

and $S_{2}(k)$ is the pair structural factor.

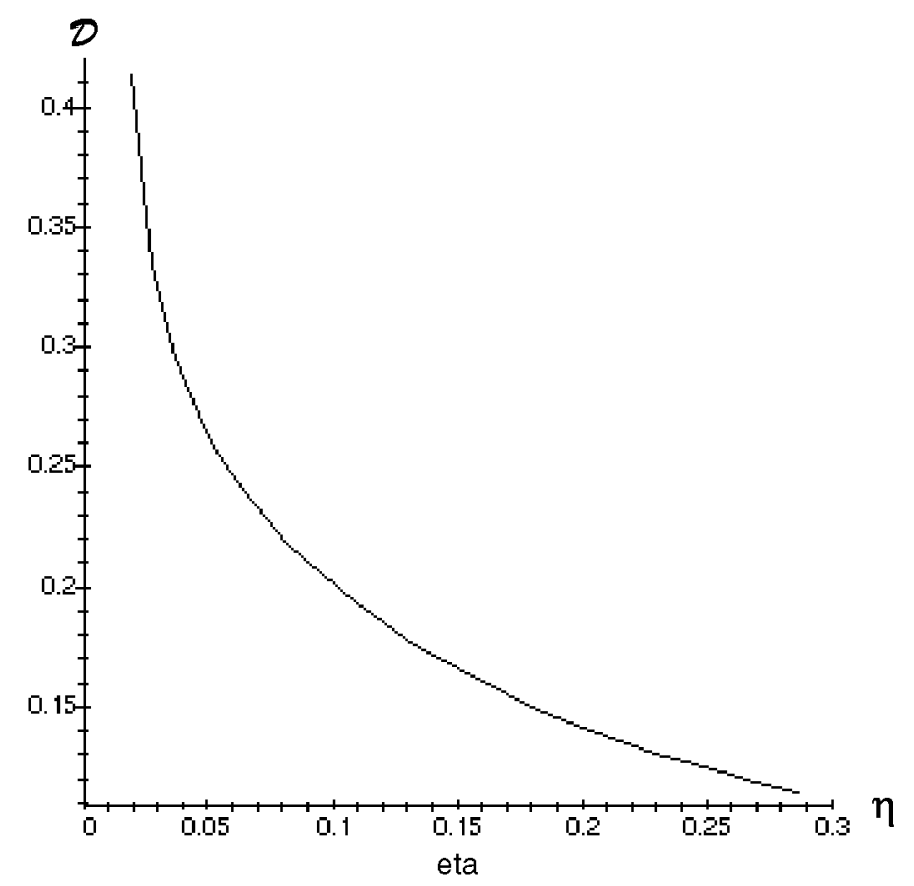

Figure 1. Dispersion distribution dependence on atoms density. 


\section{Conclusions}

The conditions are obtained at which the distribution of random values of atom levels will be of Gauss type. These conditions are:

1) Hartree-Fock approximation for Coulomb and exchange electron-electron interactions;

2) Gauss fluctuations distribution $\varrho_{\mathbf{k}}=\frac{1}{\sqrt{N}} \sum_{j=1}^{N} \mathrm{e}^{\mathrm{i} \mathbf{k} \mathbf{R}_{j}}$ of atoms density.

In this case the dispersion has the following structure

$$
\mathcal{D}=\frac{1}{V} \sum_{\mathbf{k}} f(k) S_{2}(k) .
$$

To qualitatively understand the obtained result, we depict dispersion $\mathcal{D}$ dependence on atoms density $\eta$ at fixed microscopic parameters in figure 1 . The structural factor of system $S_{2}(k)$ is in Percus-Yevick approximation for a hard core. With the increase of the density atoms, the dispersion monotonously decreases for physical reasons.

\section{References}

1. Ziman G. Models of Disorder. Cambridge University Press, 1979.

2. Klein M.W., Brout R. Statistical mechanics of dilute copper maganese. // Phys. Rev. 1963, vol. 132, No. 6, p. 2412-2426.

3. Bonch-Bruevich V.L. et al. Electronic Theory of Disorder Semiconductors. Moscow, Nauka publish., 1981.

4. Flambaum V.V., Sokolov V.V. Realistic model of correlated disorder and Anderson localisation. // Phys. Rev. B, 1999, vol. 60, No. 7, p. 4529-4531.

5. Yukhnovskii I.R., Holovko M.F. Statistical Theory of Classical Equilibrium Systems. Kyiv, Naukova dumka, 1980. 


\section{Діагональний та недіагональний типи безладу у фізиці конденсованого стану}

\section{Ю.Рудавський, Г.Понеділок, Ю.Петрів}

Національний університет "Львівська політехніка", 79013 Львів, вул. С.Бандери, 12

Отримано 28 серпня 2000 р., в остаточному вигляді 1 листопада 2000 p.

В статті досліджується функція розподілу діагонального типу безладу, її зв'язок з мікроскопічними параметрами моделі та структурними кореляційними функціями. Отримані умови, при яких розподіл випадкових величин атомних енерґетичних рівнів буде гауссового типу. Проведений чисельний аналіз дисперсії $\mathcal{D}$. Розподіл застосований до проблеми хемосорбції водневоподібних атомів на аморфній поверхні.

Ключові слова: аморфні системи, структурні функції, діагональний безлад

PACS: $73.20 . H, 82.65 . Y$ 